\title{
15 Nutzungsmöglichkeiten von SNOMED CT in der Schweiz
}

Sang-Il Kim

\subsection{Motivation für SNOMED CT}

Für eine qualitativ bessere und effizientere Patientenbehandlung ist ein optimaler Informationsaustausch zwischen den Behandelnden notwendig. Dadurch kann auch die Patientensicherheit verbessert werden. Die technische Interoperabilität der beteiligten medizinischen Informationssysteme, wie Arztpraxisinformationssysteme oder Klinikinformationssysteme, ist grundlegende Bedingung für den digitalen Austausch von Patientendaten und -dokumenten. Zusätzlich zur technischen Interoperabilität muss auch die semantische Interoperabilität gewährleistet werden, damit Sender und Empfänger einer Information auch das Gleiche verstehen. Im einfachsten Fall geschieht dies durch bilaterale Absprachen und Einigung auf ein gemeinsames Vokabular. Im Kontext der vielfältigen medizinischen Informationen werden rein bilaterale Vereinbarungen nicht ausreichen, insbesondere wenn viele tausende Akteure und Systeme miteinander Informationen digital austauschen sollen. Dafür gibt es zahlreiche internationale semantische Standards, wie zum Beispiel ICD-10, LOINC oder SNOMED CT (Systematized Nomenclature of Human and Veterinary Medicine - Clinical Terms).

Bei der Definition von Austauschformaten für das elektronische Patientendossier (EPD) sind die Grenzen von nicht-lizenzpflichtigen Terminologien und Klassifikationen wie ICD-1o oder LOINC sichtbar geworden. Immer wieder 
B Der fachliche Hintergrund: Terminologien und Ordnungssysteme in der

Patientenversorgung, medizinischen Forschung und Gesundheitswirtschaft

wurde die Notwendigkeit für eine umfassendere Terminologie/Nomenklatur erkannt, damit komplexe medizinische Sachverhalte semantisch korrekt und mit der notwendigen fachlichen Aussagekraft kodiert werden können. SNOMED CT bietet diese Möglichkeiten und ist als etablierter internationaler Standard gut geeignet für die Kodierung von medizinischen Informationen in den Austauschformaten für das EPD. Darüber hinaus kann SNOMED CT in weiteren Kontexten benutzt werden, zum Beispiel für die Kodierung von medizinischen Inhalten in Arztpraxisinformationssystemen oder Klinikinformationssystemen.

\subsection{Vereinbarung mit IHTSDO für Teilnutzung bis Ende 2015}

Im September 2013 haben „eHealth Suisse“ und das Bundesamt für Gesundheit (BAG) mit der „International Health Terminology Standards Development Organisation“ (IHTSDO) eine Vereinbarung für den schrittweisen Einstieg der Schweiz in die Nutzung von SNOMED CT abgeschlossen. Die IHTSDO mit Sitz in Kopenhagen (DK) verwaltet die SNOMED CT-Terminologie und entwickelt sie weiter. Gemäß der Vereinbarung darf die Schweiz in einer Übergangszeit von drei Jahren bis Ende 2015 SNOMED CT-Codes in folgenden von „eHealth Suisse" empfohlenen Austauschformaten einsetzen:

- „Laborbefunde im Transplantationsprozess“

- "Meldepflichtige Laborbefunde“

- Zukünftige Inhalte des „ePatientendossiers“ (z.B. „eImpfdossier“, „eMedikation“, „eAustrittsbericht“").

Die Obergrenze liegt derzeit bei 5oo Konzepten von SNOMED CT, wobei diese Obergrenze nach gegenseitiger Absprache nach oben angepasst werden kann.

Bis Ende 2015 muss ein reduzierter Lizenzbetrag bezahlt werden. Für die Zeit danach streben „eHealth Suisse“ und BAG so rasch wie möglich eine volle Mitgliedschaft an, wobei die Finanzierung der nationalen Lizenz möglichst breit abgestützt werden soll.

\subsection{Prozess für Antrag auf SNOMED CT-Codes}

„eHealth Suisse“ verwaltet die Liste der SNOMED CT-Codes, die in den verschiedenen Austauschformaten genannt werden und über die Vereinbarung abgedeckt sind. Alle aufgelisteten SNOMED CT-Codes können von allen Akteuren im Schweizer Gesundheitswesen lizenzkostenfrei genutzt werden, insbesondere betrifft dies die Hersteller von Medizin-Software-Systemen und die Anwender. Diese Liste wird auf der Webseite von „eHealth Suisse“ veröffentlicht.

Für jede weitere Nutzung von SNOMED CT-Codes bis Ende 2015 muss „eHealth Suisse" angefragt und informiert werden. Die Vereinbarung mit der IHTSDO 
lässt weitere Nutzungsmöglichkeiten offen, diese müssen mit der IHTSDO jedoch ausgehandelt werden.

Ausgenommen von dieser Beschränkung sind bisherige oder zukünftige SNOMED CT-Nutzungen im Rahmen von Forschungsprojekten. Dafür sind separate Vereinbarungen mit der IHTSDO notwendig. Ebenso ausgenommen sind Anwendungsfälle, die auf bilateralen Vereinbarungen und Einzellizenzen beruhen.

\subsection{Weitere Aktivitäten}

Gemeinsam mit dem BAG wird „eHealth Suisse“ versuchen, die notwendigen Rahmenbedingungen für eine nationale SNOMED CT-Lizenz zu schaffen (spätestens ab 2016). Dies betrifft sowohl die jährliche Finanzierung der Lizenzkosten durch private und öffentliche Mittel als auch den Aufbau von Infrastrukturen und Fachwissen für die Nutzung, Pflege und Weiterentwicklung der SNOMED CT-Terminologie. 